\title{
Using Smartphones to Estimate Road Pavement Condition
}

\author{
Viengnam Douangphachanh ${ }^{\mathrm{a}}$ \\ Hiroyuki Oneyama $^{\mathrm{a}}$
}

\begin{abstract}
Efficient road infrastructure maintenance and management depends on many factors, of which the availability of updated pavement condition data is among the most important. Today's smartphones, which usually come with many sensors, are potentially useful tools for pavement condition estimation. This paper explores the use of data from smartphones' accelerometers to analyze for features and relationship of acceleration vibration to estimate road roughness condition. Although, the estimation might not be as accurate as modern profilers, it still may be very useful for cost saving and as an indicator for continuous monitoring. In the experiment, smartphones are placed inside vehicles and drive along selected road sections to gather data for analysis. The analysis consists of data filtering, matching with location and reference data, sectioning and frequency domain analysis. Results show that acceleration vibration magnitude has a linear relationship with road roughness condition.
\end{abstract}

\section{Key words: Smartphones; Road Pavement Condition; Pavement Condition Estimation; Road Roughness}

\section{Introduction}

Road pavement condition can be defined by the irregularity, which may be in the form of surface unevenness, potholes, cracks, deterioration or damages and so forth, in the pavement surface that adversely affects the ride quality of vehicles. Road roughness is an internationally accepted indicator to which it is usually used to measure the condition of road pavement. Roughness is an important pavement characteristic because it affects not only ride quality but also vehicle delay costs, fuel consumption and maintenance costs. The International Roughness Index (IRI) is a measurement indicator that has been used internationally for road pavement condition $^{1}$.

Bad pavement condition can cause; damage to vehicles, may increase fuel consumption, increase road user costs for vehicle maintenance, reduced driving comfort, and sometimes it may pose a significant traffic safety threat to road users. Therefore, pavement condition information is usually of the interests of the general public, road users and particularly the government or road authorities. For the authorities, the information is crucial in their decision making process especially for strategic planning such as management planning, maintenance planning and programming of the road infrastructure. The lack of sufficient availability of funding, technology

${ }^{a}$ Graduate School of Civil and Environmental Engineering, Tokyo Metropolitan University, Hachioji Tokyo 192-0397, Japan, douangphachanh-viengnam@ed.tmu.ac.jp http://dx.doi.org/10.14453/isngi2013.proc.16 
and skillful manpower often leads to infrequent collection of pavement condition data, thus the data is usual left outdated. Consequently, sound management and maintenance of the road infrastructure have often been compromised, which is usually viewed as a great challenge for many road authorities in maintaining good quality of road infrastructure under budget constraint, particularly in developing countries.

Using smartphone as a tool to collect data is a promising alternative because of its low cost and easy to use features in addition to its potentially wide population coverage as probe devices. Smartphones nowadays usually come with many useful sensors. Accelerometers and GPS are among many sensors that can be found in today's smartphones. Many researchers have proposed approaches that use smartphones to detect road anomalies in the hope of increasing the ability of road authorities to collect data at an appropriate frequency, required for management and maintenance planning. Noticeably, the majority of these studies focus mainly on identifying and locating road anomalies such as bumps, potholes, cracks and so forth. One of the final aims of our research, however, is to focus on the use of smartphones to estimate pavement condition (roughness) based on IRI, which is different from the previous researches. Being able to obtain roughness condition data of the pavement by an easier mean, road authorities would understand more thoroughly about the condition of their road network. Thus, be able to make better plans for road network management, maintenance and monitoring. To achieve our final goal, this paper explores features and the relationship between smartphone sensor data, collected by accelerometer and GPS, and road roughness condition (IRI). Such information would enable us to develop a smartphone application with simple algorithms or models that can be used to evaluate or estimate road roughness condition.

\section{Related Work}

Using smartphone and mobile sensors to detect road bumps and anomalies has been studied and proposed for pavement monitoring and management purposes in recent years. Previous work that is most relevant to our research includes: the experiment conducted by Gonzalez et al. $^{2}$, through simulation, mobile accelerometers can be used to assess road roughness condition. The Pothole Patrol ${ }^{3}$, a system that also utilizes mobile accelerometers has been developed to successfully detect road anomalies. Mohan et al. ${ }^{4}$ used many sensing components from mobile phones such as accelerometers, microphones, GSM radio, and GPS to monitor road and traffic conditions. By analyzing data from the sensors, potholes, bumps, braking and honking can be detected. The information is then used to assess road and traffic conditions. In some studies ${ }^{5-6}$ Android smartphone devices with accelerometers are used to detect location of potholes. Their approach includes many simple algorithms to detect events in the acceleration vibration data. Analyzing data obtained by smartphone accelerometers in frequency domain to extract features that are corresponding to road bumps is carried out in the work of Tai et al. ${ }^{7}$ and Perttunen et al. ${ }^{8}$

\section{Methodology}

Different road pavement conditions cause passing-by vehicles to vibrate differently. We assume that the vibration can be recorded by placing smartphones, which come with accelerometers, somewhere inside the vehicle. In our experiment, we place two smartphones on four different vehicles (one vehicle at a time). Under normal driving conditions, we drive each 
experiment vehicle along many selected roads that have different pavement conditions, with condition indexes ranging from good, fair, poor, and bad, respectively based on IRI, See Table 1 below. Two different sensors on the smartphones are set to record acceleration and GPS data. To simplify our experiment, the orientation of the smartphones is fixed. Thus, we assume that the acceleration coordinates of the vehicle and smartphones are the same. A video camera is also used to capture the road surface; this video footage is used for data checking and verification if required.

A Vehicle Intelligent Monitoring System or $V I M S^{9}$ is also used to estimate condition (IRI) of the roads selected for our experiment. Results from VIMS are used as reference road roughness condition data. The experiment was conducted in Vientiane Capital City, Lao PDR, from 16 to 21 November 2012.

Table 1. Pavement Condition Index

\section{Condition Index}

Good

Fair

Poor

$\mathrm{Bad}$

\section{Average IRI}

$0 \leq \mathrm{IRI}<4$

$4 \leq \mathrm{IRI}<7$

$7 \leq \mathrm{IRI}<10$

$\mathrm{IRI} \geq 10$

\section{Data Collection}

\section{A. Equipment and Software}

The main equipment for our experiment is shown in Figure 1 below:

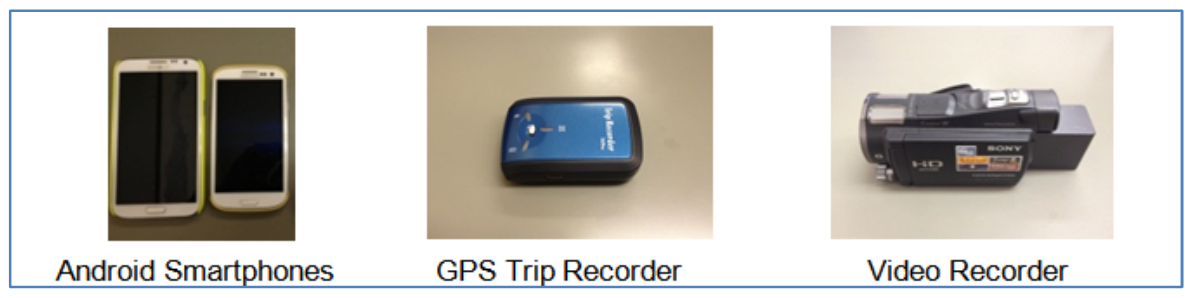

Figure 1. Experiment equipment

The experiment equipment included: two Android smartphones (a Samsung Galaxy Note 2 and a Samsung Galaxy S3), a GPS logger and a video recorder. In order to collect the required data, we installed an application called "AndroSensor", AndroSensor" ${ }^{10}$ on both smartphones. AndroSensor can collect data from almost all of the sensors available on the handsets and it is available for free download in the Google Play Store. However, for this experiment, only acceleration data $(\mathrm{x}, \mathrm{y}, \mathrm{z})$ from the accelerometer, and location data (longitude, latitude, and speed) from the GPS were needed. A recording rate of 0.01 second interval or $100 \mathrm{~Hz}$ was used.

Four Toyota vehicles, a VIGO 4WD pickup truck, a VIGO 2WD pickup truck, a Vios sedan, and a Camry sedan, were selected for our experiment. 


\section{B. Referenced Road Roughness Condition Data}

VIMS was used to obtain the referenced pavement condition data used in this study. VIMS is developed by The Bridge and Structure Laboratory at the University of Tokyo, Japan. The system is now being deployed for road management purpose in Laos. For our experiment, VIMS was used with the experiment vehicles. To use VIMS, proper calibrations are carried out, details can be found in VIMS ${ }^{11}$. VIMS data collection for the four vehicles is carried out at the same time of the smartphone data collection.

VIMS uses an accelerometer and a GPS on a vehicle. The vehicle then drives on a road and calculates the International Roughness Index (IRI) in a short time based on acceleration response of the vehicle. VIMS calculates IRI for every 10 meter road section. The result can be put in an Excel spreadsheet as well as output to a visual presentation on Google Earth. VIMS can only calculate IRI when driving speed of experiment vehicle is $20 \mathrm{kph}$ or faster.

\section{Data collection setting}

The two smartphones were glued closed to each other on the dashboard of the experiment vehicles with strong and thin adhesive tapes. The screens of the smartphones are facing up and the heads point towards the front of the vehicle. Therefore, the $\mathrm{x}, \mathrm{y}$ and $\mathrm{z}$ axes of the accelerometer represent the motion along left-right, front-rear and up-down of the vehicle, respectively. Other equipment such as GPS and video camera are also placed on the dash board. VIMS components are also installed in accordance to the VIMS manual ${ }^{11}$.

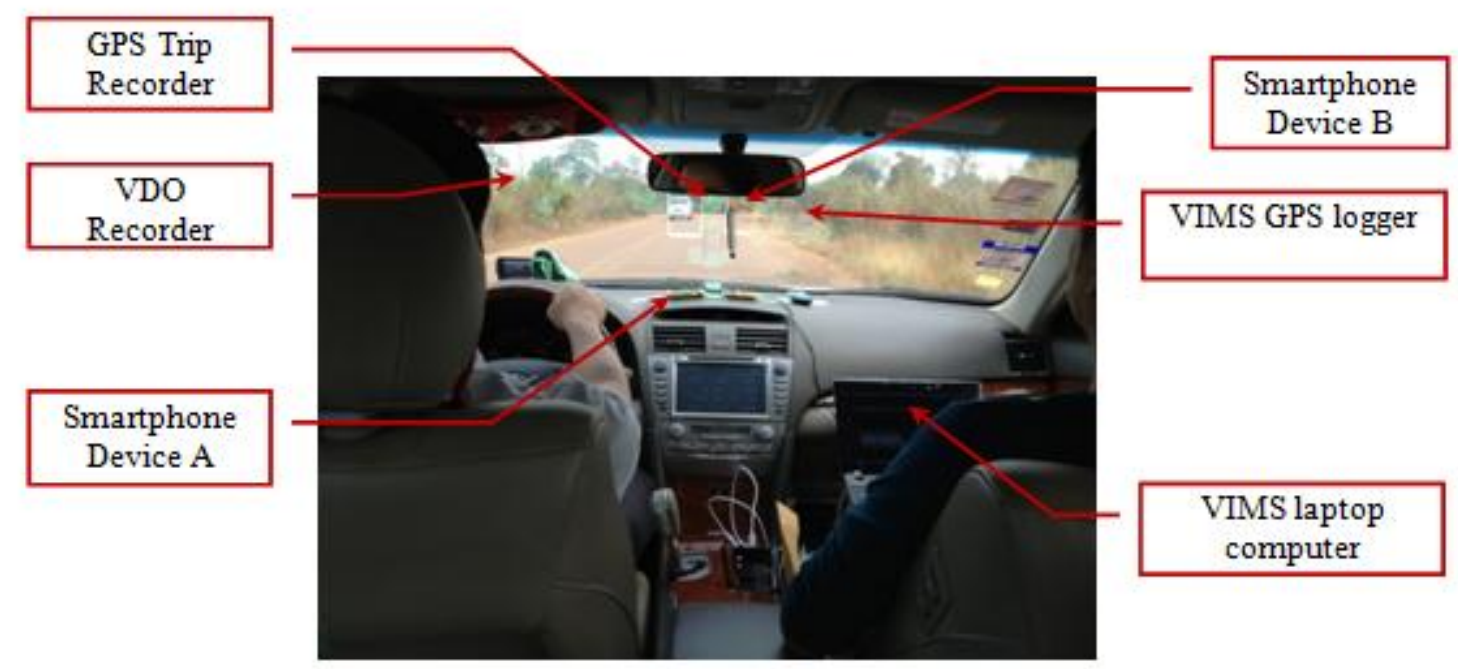

Figure 2. Data collection setting

\section{Data Processing}

After data from smartphones was uploaded onto a desktop computer and converted to excel spreadsheets, validation checks were carried out. The validation is to ensure that only road sections that have complete data sets, both data from smartphones and IRI data from VIMS, will be selected for further analysis. Sections with incomplete data are the sections that have no data 
from smartphones and/or VIMS, that is when the vehicle speed is too slow (<20kph) (i.e in heavy traffic conditions).

A high pass filter, a standard method used for Android device, Android Developer Reference $^{12}$, is applied to remove unrelated low frequency signals, which are usually caused by the effect of vehicle motion as the result of changing speed or turning, as well as the contribution of the force of gravity, from all axes ( $\mathrm{x}, \mathrm{y}$ and $\mathrm{z}$ ) on the acceleration data.

Data matching by GPS coordinates is carried out. This process merges two separate data files, IRI data from VIMS and the acceleration data from the smartphones for the same road sections based on GPS coordinates, to form a new data file. Then, the new data files are cut into small 100 meter sections. A 100 meter length of acceleration data is chosen as a unit for road surface estimation in this study. The reasons are (i) because Road Management System in Laos requires road surface condition to be estimated for every 100 meter section, therefore it would be more convenient for us to select the same unit so that it is compatible for future application; (ii) there is a concern on the accuracy of GPS position data, thus choosing a shorter section unit may cause some issues for data matching between VIMS and smartphone GPS data. In the sectioning process, road sections where the experiment vehicles stopped (checking from speed and VIMS results) are excluded as data from these sections cannot be used to estimate road roughness condition. In addition, sections that are less than 100 meters are also ignored.

All selected data sections are converted to the frequency domain and Fast Fourier Transform (FFT) was performed. Magnitude from FFT is the amplitude or strength of the associated frequency component. For a specific frequency window, we assume that the total sum of magnitudes represents the total strength of the vibration at that frequency window. Therefore, the sum of magnitudes from FFT is studied to locate features, and examine the effect and relationship that the acceleration data might have in connection with road roughness condition.

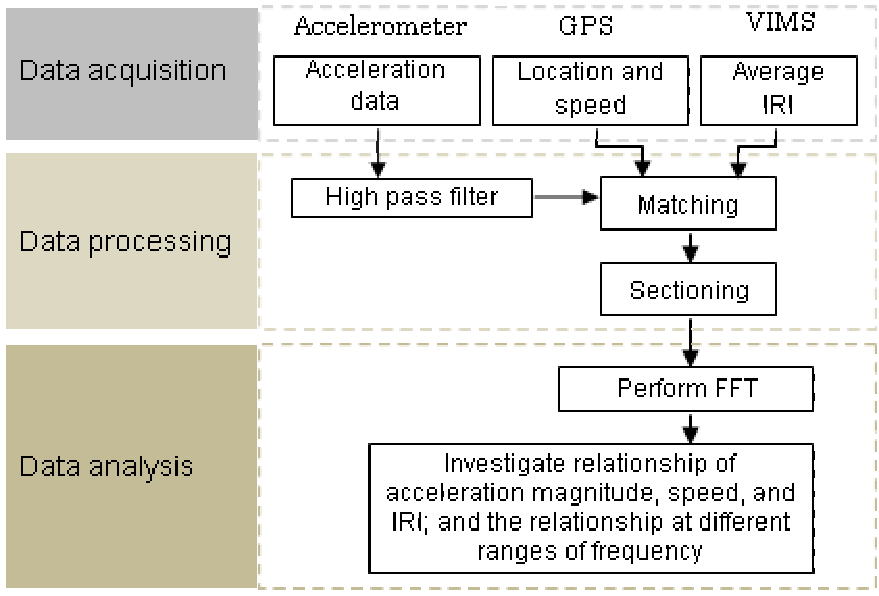

Figure 3. Data processing flow chart

\section{Analysis and Results}

From the analysis, we have found that acceleration data has linear relationship with road conditions. The correlations between the sum of magnitude of the acceleration data and average 
road roughness (IRI) for all devices and all vehicles are shown in figure 4, 5, 6 and 7 below. An average IRI is calculated from 10 VIMS IRI values (VIMS calculates IRI for every 10 meter road section) that comprises into a 100 meter road section. The sum of magnitudes is the total magnitude derived from FFT of the sum of acceleration $\mathrm{x}, \mathrm{y}$ and $\mathrm{z}$ in 100 meter road sections.

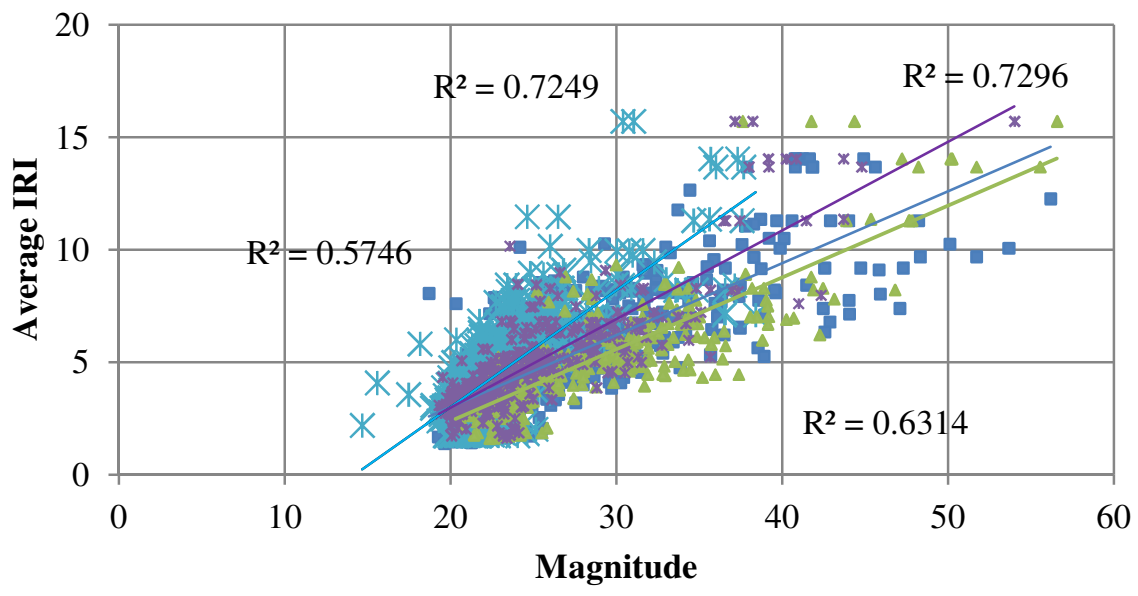

- Veh $1 *$ Veh 2 Veh $3 *$ Veh 4

Figure 4. Relationship between acceleration data and road condition (Device A, Vehicle 1, 2, 3, and 4)

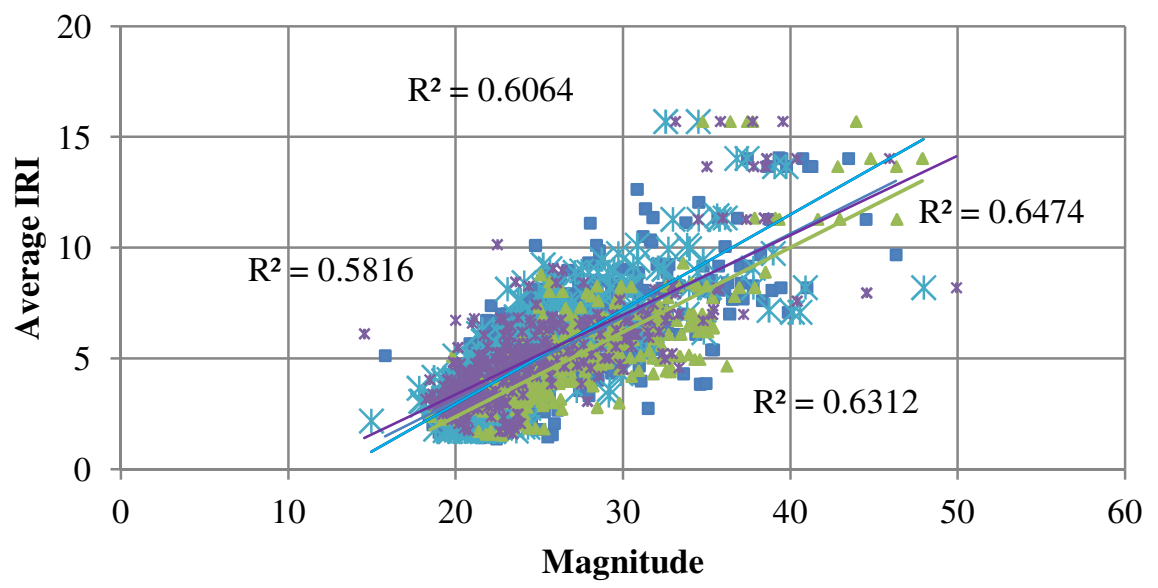

- Veh 1 *Veh 2 Veh $3 *$ Veh 4

Figure 5. Relationship between acceleration data and road condition (Device B, Vehicle 1, 2, 3, and 4) 


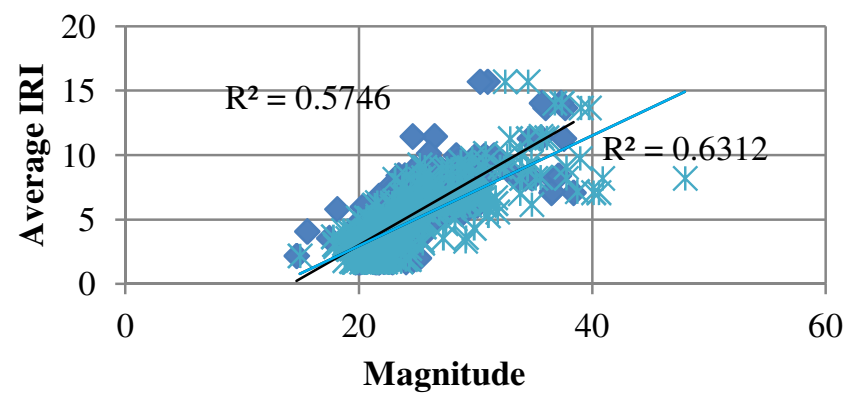

$\diamond$ Device A $*$ Device B

Figure 6. Relationship between acceleration data and road condition (Device A and B, Vehicle 2)

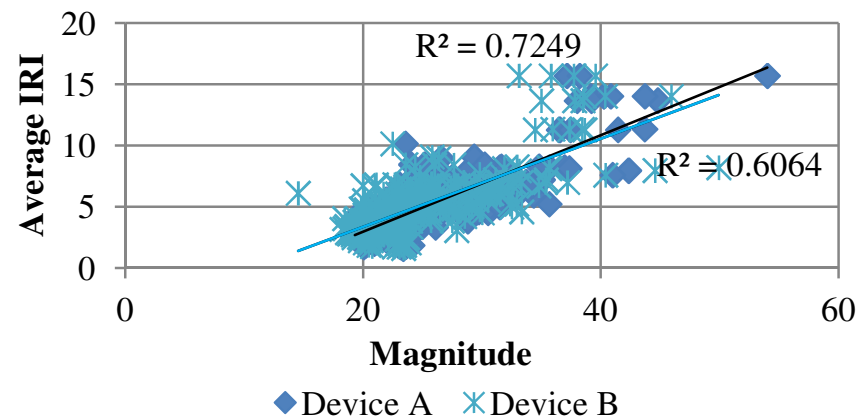

Figure 7. Relationship between acceleration data and road condition (Device A and B, Vehicle 4)

From the graphs above, it can be noted that the significant of relationship between acceleration data and road condition is slightly different between the vehicles and devices. From a statistical investigation, it is also found that the ability to estimate road roughness from acceleration data also slightly depends on the average travel speed of the vehicle.

Table 2. Summary of multiple regression analysis

\begin{tabular}{|c|c|c|c|c|c|c|c|c|}
\hline \multicolumn{9}{|c|}{ Device A } \\
\hline & \multicolumn{2}{|c|}{ Vehicle 1} & \multicolumn{2}{|c|}{ Vehicle 2} & \multicolumn{2}{|c|}{ Vehicle 3} & \multicolumn{2}{|c|}{ Vehicle 4} \\
\hline Observations & \multicolumn{2}{|c|}{703} & \multicolumn{2}{|c|}{497} & \multicolumn{2}{|c|}{314} & \multicolumn{2}{|c|}{408} \\
\hline Multiple R & \multicolumn{2}{|l|}{0.797} & \multicolumn{2}{|l|}{0.759} & \multicolumn{2}{|l|}{0.855} & \multicolumn{2}{|l|}{0.852} \\
\hline R Square & \multicolumn{2}{|l|}{0.635} & \multicolumn{2}{|l|}{0.577} & \multicolumn{2}{|l|}{0.731} & \multicolumn{2}{|l|}{0.726} \\
\hline Adjusted R Square & \multicolumn{2}{|l|}{0.634} & \multicolumn{2}{|l|}{0.575} & \multicolumn{2}{|l|}{0.729} & \multicolumn{2}{|l|}{0.725} \\
\hline F Stat & \multicolumn{2}{|c|}{609.790} & \multicolumn{2}{|c|}{336.571} & \multicolumn{2}{|c|}{421.594} & \multicolumn{2}{|c|}{537.113} \\
\hline & Coefficients & t Stat & Coefficients & t Stat & Coefficients & t Stat & Coefficients & t Stat \\
\hline Intercept & -2.467 & -5.868 & -6.476 & -8.756 & -3.484 & -5.893 & -5.651 & -9.096 \\
\hline Magnitude & 0.305 & 28.820 & 0.498 & 20.757 & 0.311 & 23.603 & 0.409 & 24.678 \\
\hline Avg. Speed & -0.013 & -2.733 & -0.010 & -1.578 & -0.007 & -1.048 & 0.008 & 1.391 \\
\hline
\end{tabular}


International Symposium for Next Generation Infrastructure

October 1-4, 2013, Wollongong, Australia

\begin{tabular}{|c|c|c|c|c|c|c|c|c|}
\hline \multicolumn{9}{|c|}{ Device B } \\
\hline & \multicolumn{2}{|c|}{ Vehicle 1} & \multicolumn{2}{|c|}{ Vehicle 2} & \multicolumn{2}{|c|}{ Vehicle 3} & \multicolumn{2}{|c|}{ Vehicle 4} \\
\hline Observations & \multicolumn{2}{|l|}{674} & \multicolumn{2}{|l|}{489} & \multicolumn{2}{|l|}{319} & \multicolumn{2}{|l|}{411} \\
\hline Multiple R & \multicolumn{2}{|l|}{0.774} & \multicolumn{2}{|l|}{0.798} & \multicolumn{2}{|l|}{0.805} & \multicolumn{2}{|l|}{0.779} \\
\hline R Square & \multicolumn{2}{|l|}{0.599} & \multicolumn{2}{|l|}{0.638} & \multicolumn{2}{|l|}{0.647} & \multicolumn{2}{|l|}{0.607} \\
\hline Adjusted R Square & \multicolumn{2}{|c|}{0.598} & \multicolumn{2}{|l|}{0.636} & \multicolumn{2}{|l|}{0.645} & \multicolumn{2}{|l|}{0.605} \\
\hline F Stat & \multicolumn{2}{|c|}{501.448} & \multicolumn{2}{|c|}{427.417} & \multicolumn{2}{|c|}{290.138} & \multicolumn{2}{|c|}{314.653} \\
\hline & Coefficients & t Stat & Coefficients & t Stat & Coefficients & $\mathrm{t}$ Stat & Coefficients & t Stat \\
\hline Intercept & -2.423 & -4.929 & -4.317 & -7.604 & -5.348 & -6.918 & -3.482 & -4.835 \\
\hline Magnitude & 0.341 & 24.595 & 0.403 & 23.684 & 0.383 & 19.531 & 0.352 & 17.879 \\
\hline Avg. Speed & -0.027 & -5.415 & -0.016 & -2.905 & 0.001 & 0.106 & -0.003 & -0.528 \\
\hline
\end{tabular}

It has been observed that the multiple regression yields a very good fitting (Figure 8). As summarized in Table 2, the intercept and coefficient of the average IRI and average speed are statistically significant. The coefficients of the average IRI is positive, meaning that the worse the road surface condition is, the larger the sum of magnitudes of vibration. On the other hand, the coefficient of the average speed is negative. This implies that a speed increase would mean a smaller sum of magnitudes, which could mean better road surface condition. One of the reasons could be, however, in general, drivers tend to drive at a higher speed on good roads; and at a much slower speed on road with bad surface condition. In addition, although the order of the coefficients is very similar for all devices and vehicle, the difference of coefficient by vehicle type and devices is observed.

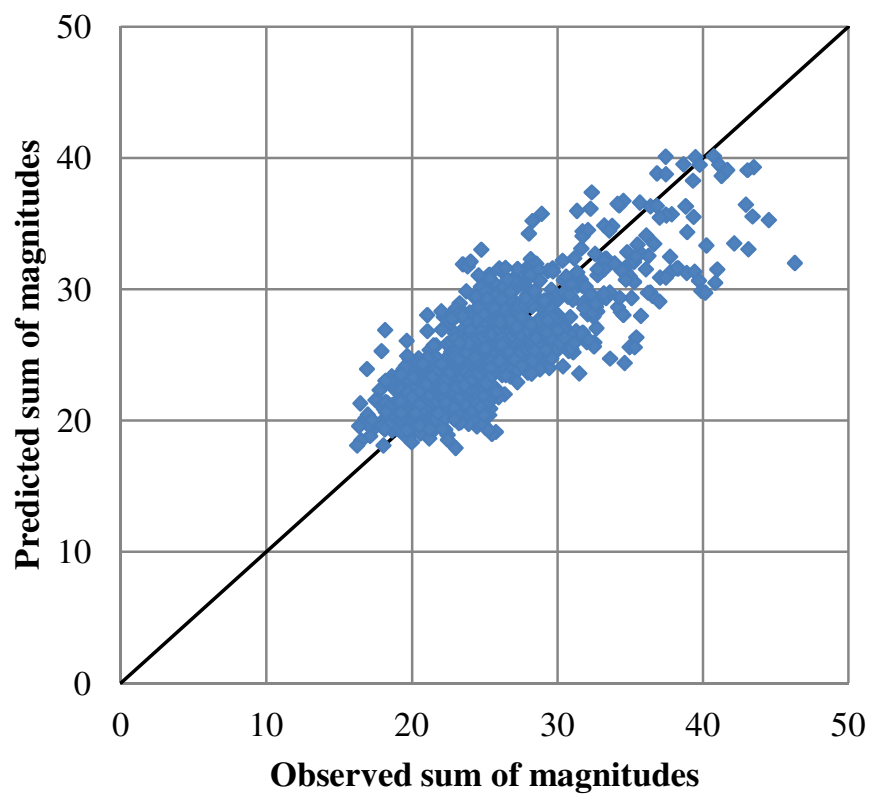

\section{Figure 8. Performance of the multiple regression model}

To sum up, from our analysis, it is clear that IRI can be expressed roughly as a linear function of magnitude of acceleration vibration and average speed of the vehicle. Parameters (coefficients) of linear function are different representing different vehicles and devices. 


\section{Conclusion}

The main objective of this paper is to explore the use of smartphones for road infrastructure management and maintenance purposes. Rough estimation of road pavement condition from smartphones would be helpful enough for such purpose, provided that the approach is low cost, easy to operate and can be implemented frequently.

After obtaining data from our experiment, processing and analysis, it has been found that acceleration data from smartphones has linear relationship with road roughness condition. However, the significant of relationship depends on the average speed of vehicles. We conclude that a simple model would be sufficient to estimate road roughness condition from acceleration data obtained by smartphones. In our future studies, more realistic setting of smartphone devices, free orientation, will be considered.

\section{References}

${ }^{1}$ Sayers, M. W., Gillespie, T. D., and Queiros, C. A. V., International Road Roughness Experiment. The World Bank, 1986, URL:

http://deepblue.lib.umich.edu/bitstream/handle/2027.42/3134/72773.pdf;jsessionid=2D55BF 78AABC A31452E6A59CD28D17C1? sequence $=2$

${ }^{2}$ González, A., O’brien, E. J., Li ,Y. Y., and Cashell, K., “ The use of vehicle acceleration measurements to estimate road roughness", Vehicle System Dynamics, Vol. 46, No. 6, 2008, pp. 483-499. http://dx.doi.org/10.1080/00423110701485050

${ }^{3}$ Eriksson, J., Girod, L., Hull, B., Newton, R., Madden, S., and Balakrishnan, H., "The pothole patrol: using a mobile sensor network for road surface monitoring", Sixth International Conference on Mobile System, Applications and Services, Breckenridge, Colorado, United States, June 17-20, 2008.

${ }^{4}$ Mohan, P., Padmanabhan, V.N., and Ramjee, R., "Nericell: Rich Monitoring of Road and Traffic Condition using Mobile Smartphones", Proceedings of the $6^{\text {th }}$ ACM Conference on Embedded Network Sensor Systems, 2008, pp. 323-336. http://dx.doi.org/10.1145/1460412.1460444

${ }^{5}$ Mednis, A., Strazdins, G., Zviedris, R., Kanonirs, G., and Selavo, L., "Real time pothole detection using Android smartphones with accelerometers", 2011 International Conference on Distributed Computing in Sensor Systems, Barcelona, Spain, June 27-29, 2011.

${ }^{6}$ Strazdins, G., Mednis, A., Kanonirs, G., Zviedris, R., and Selavo, L., "Towards Vehicular Sensor Networks with Android Smartphones for Road Surface Monitoring", $2^{\text {nd }}$ International Workshop on Networks of Cooperating Objects, Chicago, USA, April 11, 2011.

${ }^{7}$ Tai, Y., Chan, C., and Hsu, J. Y., "Automatic road anomaly detection using smart mobile device", 2010 Conference on Technologies and Applications of Artificial Intelligence, Hsinchu, Taiwan, November 18-20, 2010.

${ }^{8}$ Perttunen, M., Mazhelis, O., Cong, F., Kauppila, M., Leppänen, T., Kantola, J., Collin J., Pirttikangas, S., Haverinen, J., and Ristaniemi, T., "Distributed road surface condition monitoring using mobile phones", Ubiquitous Intelligence and Computing, 2011, pp. 64-78. 
International Symposium for Next Generation Infrastructure October 1-4, 2013, Wollongong, Australia

${ }^{9}$ Vehicle Intelligent Monitoring System, VIMS, Bridge and Structure Laboratory, the University of Tokyo, Japan, 2012.

${ }^{10}$ Android Application, AndroSensor. Google Play Store, 2012, URL: https:// play.google.com/store/ apps/details?id=com.fivasim.androsensor\&hl=en

${ }^{11}$ VIMS, VIMS Manual, VIMS Consortium, Nagasaki University, Japan, 2012.

${ }^{12}$ Android Developer Reference, SensorEvent, Android Developer Official Website, 2012, URL: http://developer.android.com/reference/android/hardware/SensorEvent.html 